\title{
Certain Subclass of $m$-Valent Functions Associated with a New Extended Ruscheweyh Operator Related to Conic Domains
}

\author{
Amnah E. Shammaky $\mathbb{D}^{1}$ and Tamer M. Seoudy $\mathbb{D}^{2,3}$ \\ ${ }^{1}$ Department of Mathematics, Faculty of Science, Jazan University, Jazan, Saudi Arabia \\ ${ }^{2}$ Department of Mathematics, Faculty of Science, Fayoum University, Fayoum 63514, Egypt \\ ${ }^{3}$ Department of Mathematics, Jamoum University College, Umm Al-Qura University, Makkah, Saudi Arabia \\ Correspondence should be addressed to Tamer M. Seoudy; tms00@fayoum.edu.eg \\ Received 6 June 2021; Accepted 7 July 2021; Published 31 July 2021 \\ Academic Editor: Sibel Yalçın
}

Copyright (c) 2021 Amnah E. Shammaky and Tamer M. Seoudy. This is an open access article distributed under the Creative Commons Attribution License, which permits unrestricted use, distribution, and reproduction in any medium, provided the original work is properly cited.

The main object of the present paper is to introduce certain subclass of $m$-valent functions associated with a new extended Ruscheweyh linear operator in the open unit disk. Also, we investigate a number of geometric properties including coefficient estimates and the Fekete-Szegö type inequalities for this subclass. Several known consequences of the main results are also pointed out.

\section{Introduction}

Let $\mathscr{A}(m)$ denote the class of functions of the next form:

$$
f(\xi)=\xi^{m}+\sum_{n=m+1}^{\infty} a_{n} \xi^{n}(m \in \mathbb{N}=\{1,2,3, \cdots\})
$$

which are analytic and $m$-valent in the open unit disc $\mathbb{D}=\{$ $\xi \in \mathbb{C}:|\xi|<1\}$, and let $\mathscr{A}(1)=\mathscr{A}$. Also, let $f, g$ be analytic in $\mathbb{D}$, and the function $f(\xi)$ is said to be subordinate to $g(\xi)$ if there exists a function $\omega(\xi)$ analytic in $\mathbb{D}$ with $\omega(0)=0$ and $|\omega(\xi)|<1, \xi \in \mathbb{D}$, such that $f(\xi)=g(\omega(\xi))$. In such a case, we write $f(\xi)<g(\xi)$. If $g$ is univalent function, then $f$ $(\xi)<g(\xi)$ if and only if $f(0)=g(0)$ and $f(\mathbb{D}) \subset g(\mathbb{D})$ (see $[1,2]$ and $[3])$.
For functions $f(\xi)$ given by $(1)$ and $g(\xi)$ is defined by

$$
g(\xi)=\xi^{m}+\sum_{n=m+1}^{\infty} b_{n} \xi^{n}
$$

and the Hadamard product or convolution of $f(\xi)$ and $g(\xi)$ is defined by

$$
(f * g)(\xi)=\xi^{m}+\sum_{n=m+1}^{\infty} a_{n} b_{n} \xi^{n}
$$

For $v \in \mathbb{C}, k \in \mathbb{R}$, and $n \in \mathbb{N}$, the Pochhammer $k$-symbol $(v)_{n, k}$ is given by (see [4])

$$
(v)_{n, k}=v(v+k)(v+2 k) \cdots(v+(n-1) k)=\prod_{i=1}^{n}(v+(i-1) k) .
$$


We define the function $\phi_{m}(\delta, k ; \xi)$ by

$$
\begin{aligned}
\phi_{m}(\delta, k ; \xi) & =\frac{\xi^{m}}{(1-\xi)^{\delta+m k / k}} \\
& =\xi^{m}+\sum_{n=m+1}^{\infty} \frac{(\delta+m k)_{n-m, k}}{(k)_{n-m, k}} \xi^{n}(\delta>-m k ; k>0 ; \xi \in \mathbb{D}) .
\end{aligned}
$$

Corresponding to the function $\phi_{m}(\delta, k ; \xi)$, we consider a linear operator $\mathscr{D}^{\delta+m k-k}: \mathscr{A}(m) \longrightarrow \mathscr{A}(m)(\delta>-m k, k>0)$ which is defined by means of the following Hadamard product (or convolution):

$\mathscr{D}^{\delta+m k-k} f(\xi)=\phi_{m}(\delta, k ; \xi) * f(\xi)=\xi^{m}+\sum_{n=m+1}^{\infty} \frac{(\delta+m k)_{n-m, k}}{(k)_{n-m, k}} a_{n} \xi^{n}(\xi \in \mathbb{D})$.

It is easily verified from (6) that

$k \xi\left(\mathscr{D}^{\delta+m k-k} f(\xi)\right)^{\prime}=(\delta+m k) \mathscr{D}^{\delta+m k} f(\xi)-\delta \mathscr{D}^{\delta+m k-k} f(\xi)(k>0)$.

We note that

(1) For $k=1$, the operator $\mathscr{D}^{\delta+m k-k} f(\xi)$ reduced to the differential operator $\mathscr{D}^{\delta+m-1} f(\xi)$ introduced by Goel and Sohi [5] (see also [6, 7] and [8])

(2) For $m=1$, we obtain the $k$-Ruscheweyh derivative operator $\mathscr{D}_{k}^{\delta}([9])$, where

$$
\mathscr{D}_{k}^{\delta} f(\xi)=\frac{\xi}{(1-\xi)^{\delta+k / k}} * f(\xi)=\xi+\sum_{n=2}^{\infty} \frac{(\delta+k)_{n-1, k}}{(k)_{n-1, k}} a_{n} \xi^{n}
$$

(3) For $k=m=1$, the operator $\mathscr{D}^{\delta+m k-k} f(\xi)$ reduced tothe well-familiar Ruscheweyh operator $\mathscr{D}^{\delta}([10])$

(4) For $\delta=k-m k$,we have $\mathscr{D}^{0} f(\xi)=f(\xi)$, and for $\delta=2$ $k-m k$, we get $\mathscr{D}^{k} f(\xi)=\xi^{m}\left(\xi^{1-m} f(\xi)\right)^{\prime}$

By using the linear operator $\mathscr{D}^{\delta+m k-k} f(\xi)$, we define the subclass $\beta-\mathcal{S} \mathscr{T}_{m}(\delta, k, b)$ of $\mathscr{A}(m)$ as follows:

Definition 1. Let $\beta \geq 0, \delta>-m k, m \in \mathbb{N}, k>0, b \in \mathbb{C}^{*}=\mathbb{C} \backslash$ $\{0\}$, and $\xi \in \mathbb{D}$. A function $f \in \mathscr{A}(m)$ is in the class $\beta-\mathcal{S}$ $\mathscr{T}_{m}(\delta, k, b)$, if it satisfies

$\Re\left\{1+\frac{1}{b}\left(\frac{\xi\left(D^{\delta+m k-k} f(\xi)\right)^{\prime}}{m \mathscr{D}^{\delta+m k-k} f(\xi)}-1\right)\right\} \quad>\beta\left|\frac{1}{b}\left(\frac{\xi\left(\mathscr{D}^{\delta+m k-k} f(\xi)\right)^{\prime}}{m \mathscr{D}^{\delta+m k-k} f(\xi)}-1\right)\right|$.

Geometrically, a function $f \in \beta-\mathcal{S} \mathscr{T}_{m}(\delta, k, b)$ if and only if

$$
1+\frac{1}{b}\left(\frac{\xi\left(\mathscr{D}^{\delta+m k-k} f(\xi)\right)^{\prime}}{m \mathscr{D}^{\delta+m k-k} f(\xi)}-1\right)
$$

takes all the values in the conic domain $\Omega_{\beta}=\psi_{\beta}(\mathbb{D})$, where

$$
\Omega_{\beta}=\left\{u+i v: u>\beta \sqrt{(u-1)^{2}+v^{2}}\right\}
$$

or equivalently,

$$
1+\frac{1}{b}\left(\frac{\xi\left(\mathscr{D}^{\delta+m k-k} f(\xi)\right)^{\prime}}{m \mathscr{D}^{\delta+m k-k} f(\xi)}-1\right) \prec \psi_{\beta}(\xi), \Omega_{\beta}=\psi_{\beta}(\mathbb{D}) .
$$

The boundary $\partial \Omega_{\beta}$ of the above set becomes the imaginary axis when $\beta=0$, a hyperbola when $0<\beta<1$, a parabola when $\beta=1$, and an ellipse when $1<\beta<\infty$. The functions $\psi_{\beta}(\xi)$ are defined by

$$
\psi_{\beta}(\xi)= \begin{cases}\frac{1+\xi}{1-\xi} & (\beta=0), \\ 1+\frac{1}{1-\beta^{2}} \cos \left(\frac{2}{\pi}\left(\cos ^{-1} \beta\right) i \log \left(\frac{1+\sqrt{\xi}}{1-\sqrt{\xi}}\right)\right) & (0<\beta<1), \\ 1+\frac{2}{\pi^{2}}\left(\log \frac{1+\sqrt{\xi}}{1-\sqrt{\xi}}\right)^{2} & (\beta=1), \\ 1+\frac{1}{\beta^{2}-1} \sin \left(\frac{\pi}{2 R(t)} \int_{0}^{u(\xi) / \sqrt{t}} \frac{d x}{\sqrt{1-x^{2}} \sqrt{1-t^{2} x^{2}}}\right)+\frac{\beta^{2}}{\beta^{2}-1} & (1<\beta<\infty),\end{cases}
$$

with $u(\xi)=\xi-\sqrt{t} / 1-\sqrt{t \xi}(0<t<1, \xi \in \mathbb{D})$, where $t$ is chosen such that $k=\cosh \left(\pi R^{\prime}(t) / 4 R(t)\right)$, and $R(t)$ is the Legendre's complete elliptic integral of the first kind and $R^{\prime}(t)$ the complementary integral of $R(t)$ (see $[11,12]$ and [13]).

By taking specific values to the parameters $\beta, m, \delta, k$, and $b$ in the subclass $\beta-\mathcal{S} \mathscr{T}_{m}(\delta, k, b)$, we obtain

(1) $\beta-\mathcal{S T}_{m}\left(\delta, k,(1-\alpha / m) \cos \gamma e^{-i \gamma}\right)=\beta-\mathcal{S F}_{m}^{\gamma}(\delta, k$,

$\alpha)(0 \leq \alpha<m ;|\gamma|<\pi / 2)=\left\{f \in \mathscr{A}(m): e^{i \gamma} \xi\right.$ $\left(\mathscr{D}^{\delta+m k-k} f(\xi)\right)^{\prime} / \mathscr{D}^{\delta+m k-k} f(\xi) \prec(m-\alpha) \cos \gamma \psi_{\beta}(\xi)+$ $\alpha \cos \gamma+i m \sin \gamma\}$ and $\beta-\delta \mathscr{T}_{m}(\delta, k, 1-\alpha / m)=\beta$ $-\delta \mathscr{T}_{m}(\delta, k, \alpha)(0 \leq \alpha<m)=\{f \in \mathscr{A}(m): 1 / m-\alpha(\xi$ $\left.\left.\left(\mathscr{D}^{\delta+m k-k} f(\xi)\right)^{\prime} / \mathscr{D}^{\delta+m k-k} f(\xi)-\alpha\right) \prec \psi_{\beta}(\xi)\right\}$

(2) $\beta-\mathcal{S} \mathscr{T}_{m}(\delta, 1, b)=\beta-\mathcal{S} \mathscr{T}_{m}(\delta, b)=\{f \in \mathscr{A}(m): 1$ $\left.+1 / b\left(\xi\left(\mathscr{D}^{\delta+m-1} f(\xi)\right)^{\prime} / m \mathscr{D}^{\delta+m-1} f(\xi)-1\right) \prec \psi_{\beta}(\xi)\right\}$,

$\beta-\delta \mathscr{T}_{m}\left(\delta, 1,(1-\alpha / m) \cos \gamma e^{-i \gamma}\right)=\beta-\mathcal{S} \mathscr{T}_{m}^{\gamma}(\delta, \alpha)$ 
$(0 \leq \alpha<m ;|\gamma|<\pi / 2)=\left\{f \in \mathscr{A}(m): e^{i \gamma} \xi\right.$

$\left(\mathscr{D}^{\delta+m-1} f(\xi)\right)^{\prime} / \mathscr{D}^{\delta+m-1} f(\xi) \prec(m-\alpha) \cos \gamma \psi_{\beta}(\xi)+\alpha$ $\cos \gamma+i m \sin \gamma\}$ and $\beta-\delta \mathscr{T}_{m}(\delta, 1,1-\alpha / m)=\beta$ $-\delta \mathscr{T}_{m}(\delta, \alpha)(0 \leq \alpha<m)=\{f \in \mathscr{A}(m): 1 / m-\alpha(\xi$ $\left.\left.\left(\mathscr{D}^{\delta+m-1} f(\xi)\right)^{\prime} / \mathscr{D}^{\delta+m-1} f(\xi)-\alpha\right) \prec \psi_{\beta}(\xi)\right\}$

(3) $\beta-\mathcal{S T}_{1}(\delta, 1, b)=\beta-\mathcal{S T}(\delta, b)=\{f \in \mathscr{A}: 1+1 / b($ $\left.\left.\xi\left(\mathscr{D}^{\delta} f(\xi)\right)^{\prime} / \mathscr{D}^{\delta} f(\xi)-1\right) \prec \psi_{\beta}(\xi)\right\}$,

$\beta-\mathcal{S T}_{1}\left(\delta, 1,(1-\alpha) \cos \gamma e^{-i \gamma}\right)=\beta-\delta \mathscr{T}^{\gamma}(\delta, \alpha)(0$ $\leq \alpha<1 ;|\gamma|<\pi / 2)=\left\{f \in \mathscr{A}: e^{i \gamma} \xi\left(\mathscr{D}^{\delta} f(\xi)\right)^{\prime} / \mathscr{D}^{\delta} f(\xi)\right.$ $\left.\prec(1-\alpha) \cos \gamma \psi_{\beta}(\xi)+\alpha \cos \gamma+i \sin \gamma\right\}$ and $\beta-\mathcal{S}$ $\mathscr{T}_{1}(\delta, 1,1-\alpha / m)=\beta-\mathcal{S} \mathscr{T}(\delta, \alpha)(0 \leq \alpha<1)=\{f \in$ $\left.\mathscr{A}: 1 / 1-\alpha\left(\xi\left(\mathscr{D}^{\delta} f(\xi)\right)^{\prime} / \mathscr{D}^{\delta} f(\xi)-\alpha\right) \prec \psi_{\beta}(\xi)\right\}$

(4) $\beta-\mathcal{S T}_{1}(\delta, k, b)=\beta-\mathcal{S} \mathscr{T}(\delta, k, b)=\{f \in \mathscr{A}: 1+1 /$ $\left.b\left(\xi\left(\mathscr{D}_{k}^{\delta} f(\xi)\right)^{\prime} / \mathscr{D}_{k}^{\delta} f(\xi)-1\right) \prec \psi_{\beta}(\xi)\right\}$,

$\beta-\delta \mathscr{T}_{1}\left(\delta, k,(1-\alpha) \cos \gamma e^{-i \gamma}\right)=\beta-\mathcal{S F}^{\gamma}(\delta, k, \alpha)($ $0 \leq \alpha<1 ;|\gamma|<\pi / 2)=\left\{f \in \mathscr{A}: e^{i \gamma} \xi\left(\mathscr{D}_{k}^{\delta} f(\xi)\right)^{\prime} / \mathscr{D}_{k}^{\delta} f(\xi\right.$ )$\left.\prec(1-\alpha) \cos \gamma \psi_{\beta}(\xi)+\alpha \cos \gamma+i \sin \gamma\right\}$ and $\beta-\mathcal{S}$ $\mathscr{T}_{1}(\delta, k, 1-\alpha / m)=\beta-\mathcal{S} \mathscr{T}(\delta, k, \alpha)(0 \leq \alpha<1)=\{f$ $\left.\in \mathscr{A}: 1 / 1-\alpha\left(\xi\left(\mathscr{D}_{k}^{\delta} f(\xi)\right)^{\prime} / \mathscr{D}_{k}^{\delta} f(\xi)-\alpha\right) \prec \psi_{\beta}(\xi)\right\}$

(5) $\beta-\mathcal{S} \mathscr{T}_{m}(k-m k, k, b)=\beta-\mathcal{S} \mathscr{T}_{m}(b)=\{f \in \mathscr{A}(m)$ : $\left.1+1 / b\left(\xi f^{\prime}(\xi) / m f(\xi)-1\right) \prec \psi_{\beta}(\xi)\right\}$

$\beta-S \mathscr{T}_{m}\left(k-m k, k,(1-\alpha / m) \cos \gamma e^{-i \gamma}\right)=\beta-\mathcal{S} \mathscr{T}_{m}^{\gamma}$ $(\alpha)(0 \leq \alpha<m ;|\gamma|<\pi / 2)=\left\{f \in \mathscr{A}(m): e^{i \gamma} \xi f^{\prime}(\xi) / f(\xi\right.$ )$\left.\prec(m-\alpha) \cos \gamma \psi_{\beta}(\xi)+\alpha \cos \gamma+i m \sin \gamma\right\}$ and (see [14])

(6) $\beta-\mathcal{S T}_{m}(k-m k, k, 1-\alpha / m)=\beta-\mathcal{S} \mathscr{T}_{m}(\alpha)(0 \leq \alpha$ $<m)=\left\{f \in \mathscr{A}(m): 1 / m-\alpha\left(\xi f^{\prime}(\xi) / f(\xi)-\alpha\right) \prec \psi_{\beta}(\xi\right.$ )$\}$;

$\beta-\mathcal{S T}_{1}(0, k, b)=\beta-\mathcal{S} \mathscr{T}(b)=\{f \in \mathscr{A}: 1+1 / b(\xi f$ $\left.\left.{ }^{\prime}(\xi) / f(\xi)-1\right) \prec \psi_{\beta}(\xi)\right\}$,

$\beta-\mathcal{S T}_{1}\left(0, k,(1-\alpha) \cos \gamma e^{-i \gamma}\right)=\beta-\mathcal{S T}^{\gamma}(\alpha)(0 \leq \alpha$ $<1 ;|\gamma|<\pi / 2)=\left\{f \in \mathscr{A}(m): e^{i \gamma} \xi f^{\prime}(\xi) / f(\xi) \prec(1-\alpha\right.$ ) $\left.\cos \gamma \psi_{\beta}(\xi)+\alpha \cos \gamma+i \sin \gamma\right\}$ and (see [15]) $\beta-$ $\mathcal{S T}_{1}(0, k, 1-\alpha)=\beta-\mathcal{S} \mathscr{T}(\alpha)(0 \leq \alpha<1)=\{f \in \mathscr{A}$ $\left.: 1 / 1-\alpha\left(\xi f^{\prime}(\xi) / f(\xi)-\alpha\right) \prec \psi_{\beta}(\xi)\right\}$

(7) $0-\mathcal{S T}_{m}(\delta, k, b)=\mathcal{S}_{m}(\delta, k, b)=\{f \in \mathscr{A}(m): \mathfrak{R}[m+$ $\left.\left.1 / b\left(\xi\left(\mathscr{D}^{\delta+m k-k} f(\xi)\right)^{\prime} / \mathscr{D}^{\delta+m k-k} f(\xi)-m\right)\right]>0\right\}$,

$0-\delta \mathscr{T}_{m}\left(\delta, k,(1-\alpha / m) \cos \gamma e^{-i \gamma}\right)=\mathcal{S}_{m}^{\gamma}(\delta, k, \alpha)(0$ $\leq \alpha<m ;|\gamma|<\pi / 2)=\left\{f \in \mathscr{A}(m): \mathfrak{R}\left(e^{i \gamma} \xi\right.\right.$

$\left.\left.\left(\mathscr{D}^{\delta+m k-k} f(\xi)\right)^{\prime} / \mathscr{D}^{\delta+m k-k} f(\xi)\right)>\alpha \cos \gamma\right\}$, (see [16] and [17]) $0-\mathcal{S T}_{m}(k-m k, k, b)=\mathcal{S}_{m}^{\gamma}(\alpha)(0 \leq \alpha<m$ $;|\gamma|<\pi / 2)=\left\{f \in \mathscr{A}(m): \mathfrak{R}\left(e^{i \gamma} \xi f^{\prime}(\xi) / f(\xi)\right)>\alpha \cos \right.$ $\gamma\}$,(see [18]) $0-\mathcal{S T}_{m}(k-m k, k, b)=\mathcal{S}_{m}(b)=\{f \in$ $\left.\mathscr{A}(m): \mathfrak{R}\left[m+1 / b\left(\xi f^{\prime}(\xi) / f(\xi)-m\right)\right]>0\right\}$ and $\mathcal{S}_{1}(b)$ $=\mathcal{S}(b)($ see $[19,20])$

In order to establish our main results, we need the following lemmas.

Lemma 2 [21]. Let $\psi_{\beta}(\xi)(0 \leq \beta<\infty)$ be defined by (13). If

$$
\psi_{\beta}(\xi)=1+L_{1} \xi+L_{2} \xi^{2}+\cdots
$$

then

$$
L_{1}= \begin{cases}\frac{2 A^{2}}{1-\beta^{2}} & (0 \leq \beta<1), \\ \frac{8}{\pi^{2}} & (\beta=1), \\ \frac{\pi^{2}}{4 \sqrt{t}\left(\beta^{2}-1\right) R^{2}(t)(1+t)} & (1<\beta<\infty),\end{cases}
$$

$$
L_{2}= \begin{cases}\frac{A^{2}+2}{3} L_{1} & (0 \leq \beta<1), \\ \frac{2}{3} L_{1} & (\beta=1), \\ \frac{4 R^{2}(t)\left(t^{2}+6 t+1\right)-\pi^{2}}{24 \sqrt{t} R^{2}(t)(1+t)} L_{1} & (1 \leq \beta<\infty),\end{cases}
$$

where

$$
A=\frac{2 \cos ^{-1} \beta}{\pi}
$$

and $t \in(0,1)$ is chosen such that $\beta=\cosh \left(\pi R^{\prime}(t) / R(t)\right)$, and $R(t)$ is the Legendre's complete elliptic integral of the first kind.

Lemma 3 [22]. Let $h(\xi)=1+\sum_{n=1}^{\infty} c_{n} \xi^{n} \in \mathscr{P}$, i.e., let $h$ be analytic in $\mathbb{D}$ and satisfy $\mathfrak{R}\{h(\xi)\}>0$ for $\xi$ in $\mathbb{D}$; then, the following sharp estimate holds

$$
\left|c_{2}-v c_{1}^{2}\right| \leq 2 \max \{1,|2 v-1|\} \text { for all } v \in \mathbb{C} .
$$

The result is sharp for the functions given by

$$
g(\xi)=\frac{1+\xi^{2}}{1-\xi^{2}} \operatorname{org}(\xi)=\frac{1+\xi}{1-\xi}
$$


Lemma 4 [22]. If $h(\xi)=1+\sum_{n=1}^{\infty} c_{n} \xi^{n} \in \mathscr{P}$, then

$$
\left|c_{2}-v c_{1}^{2}\right| \leq \begin{cases}-4 v+2 & \text { if } v \leq 0 \\ 2 & \text { if } 0 \leq v \leq 1 \\ 4 v-2 & \text { if } v \geq 1\end{cases}
$$

and when $v<0$ or $v>1$, the equality holds if and only if $h(\xi$ )$=(1+\xi) /(1-\xi)$ or one of its rotations. If $0<v<1$, then the equality holds if and only if $h(\xi)=\left(1+\xi^{2}\right) /\left(1-\xi^{2}\right)$ or one of its rotations. If $v=0$, the equality holds if and only if

$$
h(\xi)=\left(\frac{1+\lambda}{2}\right) \frac{1+\xi}{1-\xi}+\left(\frac{1-\lambda}{2}\right) \frac{1-\xi}{1+\xi}(0 \leq \lambda \leq 1),
$$

or one of its rotations. If $v=1$, the equality holds if and only if $g$ is the reciprocal of one of the functions such that equality holds in the case of $v=0$.

Also, the above upper bound is sharp, and it can be improved as follows when $0<v<1$ :

$$
\begin{gathered}
\left|c_{2}-v c_{1}^{2}\right|+v\left|c_{1}\right|^{2} \leq 2\left(0 \leq v \leq \frac{1}{2}\right), \\
\left|c_{2}-v c_{1}^{2}\right|+(1-v)\left|c_{1}\right|^{2} \leq 2\left(\frac{1}{2} \leq v \leq 1\right) .
\end{gathered}
$$

In this paper, we investigate a coefficient estimates and the familiar Fekete-Szegö type inequalities for the subclass $\beta-\delta \mathscr{T}_{m}(\delta, k, b)$.

\section{Main Results}

We will assume throughout our discussion, unless otherwise stated, that $0 \leq \beta<\infty, m \in \mathbb{N}, \delta>-m k, k>0, b \in \mathbb{C}^{*}, L_{1}$ is given by (15), $L_{2}$ is given by (16), and $\xi \in \mathbb{D}$.

Theorem 5. Let $f \in \mathscr{A}(m)$ be given by (1). If the inequality

$$
\sum_{n=m+1}^{\infty}\{(\beta+1)(n-m)+m|b|\} \frac{(\delta+m k)_{n-m, k}}{(k)_{n-m, k}}\left|a_{n}\right| \leq m|b|,
$$

holds, then the function $f \in \beta-\delta \mathcal{T}_{m}(\delta, k, b)$.

Proof. Suppose the inequality (23) holds. Also, let us assume

$$
H(\xi)=1+\frac{1}{b}\left(\frac{\xi\left(\mathscr{D}^{\delta+m k-k} f(\xi)\right)^{\prime}}{m \mathscr{D}^{\delta+m k-k} f(\xi)}-1\right)
$$

We have

$$
\begin{aligned}
|H(\xi)-1| & =\left|\frac{1}{b}\left(\frac{\xi\left(\mathscr{D}^{\delta+m k-k} f(\xi)\right)^{\prime}}{m \mathscr{D}^{\delta+m k-k} f(\xi)}-1\right)\right| \\
& =\frac{1}{m|b|}\left|\left(\frac{\sum_{n=m+1}^{\infty}(n-m)\left((\delta+m k)_{n-m, k} /(k)_{n-m, k}\right) a_{n} \xi^{n-m}}{1+\sum_{n=m+1}^{\infty}\left((\delta+m k)_{n-m, k} /(k)_{n-m, k}\right) a_{n} \xi^{n-m}}\right)\right| \\
& \leq \frac{\sum_{n=m+1}^{\infty}(n-m)\left((\delta+m k)_{n-m, k} /(k)_{n-m, k}\right)\left|a_{n}\right|}{m|b|\left[1-\sum_{n=m+1}^{\infty}\left((\delta+m k)_{n-m, k} /(k)_{n-m, k}\right)\left|a_{n}\right|\right]} .
\end{aligned}
$$

Now consider

$$
\begin{aligned}
& \beta|H(\xi)-1|-\mathfrak{R}\{H(\xi)-1\} \\
& \quad \leq(\beta+1)|H(\xi)-1| \\
& \quad<\frac{(\beta+1) \sum_{n=m+1}^{\infty}(n-m)\left((\delta+m k)_{n-m, k} /(k)_{n-m, k}\right)\left|a_{n}\right|}{m|b|\left[1-\sum_{n=m+1}^{\infty}\left((\delta+m k)_{n-m, k} /(k)_{n-m, k}\right)\left|a_{n}\right|\right]}
\end{aligned}
$$

The last expression is bounded by 1 if (23) holds. This completes the proof of Theorem 5 .

Corollary 6. If $f(\xi) \in \beta-\delta \mathscr{T}_{m}(\delta, k, b)$, then

$$
\left|a_{n}\right| \leq \frac{m|b|(k)_{n-m, k}}{\{(\beta+1)(n-m)+m|b|\}(\delta+m k)_{n-m, k}}(n \geq m+1) .
$$

The result is sharp for the function

$f(\xi)=\xi^{m}+\frac{m|b|(k)_{n-m, k}}{\{(\beta+1)(n-m)+m|b|\}(\delta+m k)_{n-m, k}} \xi^{n}(n \geq m+1)$.

Putting $m=1$ in Theorem 5, we obtain the following corollary.

Corollary 7. Let $f \in \mathscr{A}$ be given by (1) with $m=1$. If the inequality

$$
\sum_{n=2}^{\infty}\{(\beta+1)(n-1)+|b|\} \frac{(\delta+k)_{n-1, k}}{(k)_{n-1, k}}\left|a_{n}\right| \leq|b|,
$$

holds, then the function $f \in \beta-\mathcal{S T}(\delta, k, b)$.

Theorem 8. If $f \in \beta-\mathcal{S} \mathscr{T}_{m}(\delta, k, b)$, then

$$
\left|a_{m+1}\right| \leq \frac{m|b| L_{1} k}{\delta+m k}
$$

and for all $n=3,4,5, \cdots$,

$$
\left|a_{n+m-1}\right| \leq \frac{m|b| L_{1}(k)_{n-1, k}}{(n-1)(\delta+m k)_{n-1, k}} \prod_{j=1}^{n-2}\left(1+\frac{m|b| L_{1}}{j}\right) .
$$


Proof. Let

$$
g(\xi)=1+\frac{1}{b}\left(\frac{\xi\left(\mathscr{D}^{\delta+m k-k} f(\xi)\right)^{\prime}}{m \mathscr{D}^{\delta+m k-k} f(\xi)}-1\right)
$$

where

$$
g(\xi)=1+\sum_{n=1}^{\infty} c_{n} \xi^{n}
$$

is analytic function in $\mathbb{D}$, and it can be written as

$$
\sum_{n=m+1}^{\infty}(n-m) \frac{(\delta+m k)_{n-m, k}}{(k)_{n-m, k}} a_{n} \xi^{n}=m b \mathscr{D}^{\delta+m k-k} f(\xi)\left(\sum_{n=1}^{\infty} c_{n} \xi^{n}\right)
$$

Comparing the coefficients of $\xi^{n+m-1}$ on both sides

$$
\begin{aligned}
& (n-1) \frac{(\delta+m k)_{n-1, k}}{(k)_{n-1, k}} a_{n+m-1} \\
& \quad=m b\left[c_{n-1}+\frac{(\delta+m k)_{1, k}}{(k)_{1, k}} c_{n-2} a_{m+1}+\cdots+\frac{(\delta+m k)_{n-2, k}}{(k)_{n-2, k}} c_{1} a_{n+m-2}\right] .
\end{aligned}
$$

Taking absolute on both sides and then applying the coefficient estimates $\left|c_{n}\right| \leq L_{1}$ (see [13]), we have

$$
\begin{aligned}
\left|a_{n+m-1}\right| \leq & \frac{m|b| L_{1}(k)_{n-1, k}}{(n-1)(\delta+m k)_{n-1, k}} \\
& \cdot\left\{1+\frac{(\delta+m k)_{1, k}}{(k)_{1, k}}\left|a_{m+1}\right|+\cdots+\frac{(\delta+m k)_{n-2, k}}{(k)_{n-2, k}}\left|a_{n+m-2}\right|\right\} .
\end{aligned}
$$

We apply the mathematical induction on (36) so for $n=2$

$$
\left|a_{m+1}\right| \leq \frac{m|b| L_{1}(k)_{1, k}}{(\delta+m k)_{1, k}}=\frac{m|b| L_{1} k}{\delta+m k}
$$

and this shows that result is true for $n=2$. Now for $n=3$,

$$
\left|a_{m+2}\right| \leq \frac{m|b| L_{1}(k)_{2, k}}{2(\delta+m k)_{2, k}}\left\{1+\frac{(\delta+m k)_{1, k}}{(k)_{1, k}}\left|a_{m+1}\right|\right\}
$$

and using (37), we obtain

$$
\left|a_{m+2}\right| \leq \frac{m|b| L_{1}(k)_{2, k}}{2(\delta+m k)_{2, k}}\left(1+m|b| L_{1}\right)
$$

which is true for $n=3$. Let us assume that (31) is true for $n=t$ , that is,

$$
\left|a_{t+m-1}\right| \leq \frac{m|b| L_{1}(k)_{t-1, k}}{(t-1)(\delta+m k)_{t-1, k}} \prod_{j=1}^{n-2}\left(1+\frac{m|b| L_{1}}{j}\right)
$$

Consider

$$
\begin{aligned}
\left|a_{t+m}\right| \leq & \frac{m|b| L_{1}(k)_{t, k}}{t(\delta+m k)_{t, k}}\left\{1+\frac{(\delta+m k)_{1, k}}{(k)_{1, k}}\left|a_{m+1}\right|+\cdots+\frac{(\delta+m k)_{t-1, k}}{(k)_{t-1, k}}\left|a_{t+m-1}\right|\right\} \\
\leq & \frac{m|b| L_{1}(k)_{t, k}}{t(\delta+m k)_{t, k}}\left\{1+m|b| L_{1}+\cdots+\frac{(\delta+m k)_{t-1, k}}{(k)_{t-1, k}}\left|a_{t+m-1}\right|\right\} \\
\leq & \frac{m|b| L_{1}(k)_{t, k}}{t(\delta+m k)_{t, k}}\left\{1+m|b| L_{1}+\frac{m|b| L_{1}}{2}\left(1+m|b| L_{1}\right)+\cdots+\frac{m|b| L_{1}}{t-1}\right. \\
& \left.\cdot\left(1+m|b| L_{1}\right)\left(1+\frac{m|b| L_{1}}{2}\right) \cdots\left(1+\frac{m|b| L_{1}}{t-2}\right)\right\} \\
= & \frac{m|b| L_{1}(k)_{t, k}}{t(\delta+m k)_{t, k}}\left(1+m|b| L_{1}\right)\left(1+\frac{m|b| L_{1}}{2}\right) \cdots\left(1+\frac{m|b| L_{1}}{t-1}\right) \\
= & \frac{m|b| L_{1}(k)_{t, k}}{t(\delta+m k)_{t, k}} \prod_{j=1}^{t-1}\left(1+\frac{m|b| L_{1}}{j}\right) .
\end{aligned}
$$

Therefore, the result is true for $n=t+1$. Consequently, using mathematical induction, we proved that the result holds true for all $n(n \geq 2)$. This completes the proof of Theorem 8 .

Putting $m=1$ in Theorem 8 , we obtain the following corollary.

Corollary 9. If $f \in \beta-\mathcal{S T}(\delta, k, b)$, then

$$
\left|a_{2}\right| \leq \frac{|b| L_{1} k}{\delta+k}
$$

and for all $n=3,4,5, \cdots$,

$$
\left|a_{n}\right| \leq \frac{|b| L_{1}(k)_{n-1, k}}{(n-1)(\delta+k)_{n-1, k}} \prod_{j=1}^{n-2}\left(1+\frac{|b| L_{1}}{j}\right) .
$$

Theorem 10. Let $f \in \beta-\mathcal{S} \mathscr{T}_{m}(\delta, k, b)$. Then., $f(\mathbb{D})$ contains an open disk of radius

$$
r=\frac{\delta+m k}{(m+1)(\delta+m k)+m|b| L_{1} k} .
$$

Proof. Let $w_{0} \neq 0$ be a complex number such that $f(\xi) \neq w_{0}$ for $\xi \in \mathbb{D}$. Then, $f_{1}(\xi)=w_{0} f(\xi) / w_{0}-f(\xi)=\xi^{m}+\left(a_{m+1}+1 /\right.$ $\left.w_{0}\right) \xi^{m+1}+\cdots$.

Since $f_{1}$ is univalent, so

$$
\left|a_{m+1}+\frac{1}{w_{0}}\right| \leq m+1
$$

Now using Theorem 8 , we have

$$
\left|\frac{1}{w_{0}}\right| \leq m+1+\frac{m|b| L_{1} k}{\delta+m k},
$$


and hence

$$
\left|w_{0}\right| \geq \frac{\delta+m k}{(m+1)(\delta+m k)+m|b| L_{1} k}
$$

This completes the proof of Theorem 10 .

Putting $m=1$ in Theorem 10, we obtain the following corollary.

Corollary 11. Let $f \in \beta-\mathcal{S T}(\delta, k, b)$. Then, $f(\mathbb{D})$ contains an open disk of radius

$$
r_{1}=\frac{\delta+k}{2(\delta+k)+|b| L_{1} k} .
$$

Theorem 12. Let $f \in \beta-\mathcal{S} \mathscr{T}_{m}(\delta, k, b)$ with the form (1). Then, for a complex number $\mu$, we have

$\left|a_{m+2}-\mu a_{m+1}^{2}\right| \leq \frac{m b L_{1} k^{2}}{(\delta+m k)(\delta+m k+k)} \max \left\{1,\left|\frac{L_{2}}{L_{1}}+m b L_{1}\left(1-\frac{\delta+m k+k}{\delta+m k} \mu\right)\right|\right\}$.

Proof. If $f \in \beta-\delta \mathscr{T}_{m}(\delta, k, b)$, then there exists a Schwarz function $w$, with $w(0)=0$ and $|w(\xi)|<1$ such that

$$
1+\frac{1}{b}\left(\frac{\xi\left(\mathscr{D}^{\delta+m k-k} f(\xi)\right)^{\prime}}{m \mathscr{D}^{\delta+m k-k} f(\xi)}-1\right)=\psi_{\beta}(w(\xi))(\xi \in \mathbb{D})
$$

Let $h \in \mathscr{P}$ be a function defined by

$$
h(\xi)=\frac{1+w(\xi)}{1-w(\xi)}=1+c_{1} \xi+c_{2} \xi^{2}+\cdots(\xi \in \mathbb{D}) .
$$

This gives

$$
w(\xi)=\frac{c_{1}}{2} \xi+\frac{1}{2}\left(c_{2}-\frac{c_{1}^{2}}{2}\right) \xi^{2}+\cdots
$$

$\psi_{\beta}(w(\xi))=1+\frac{1}{2} c_{1} L_{1} \xi+\frac{1}{2}\left\{\frac{c_{1}^{2} L_{2}}{2}+\left(c_{2}-\frac{c_{1}^{2}}{2}\right) L_{1}\right\} \xi^{2}+\cdots$

Using (53) in (50), we obtain

$$
\begin{gathered}
a_{m+1}=\frac{m b c_{1} L_{1} k}{2(\delta+m k)}, \\
a_{m+2}=\frac{m b k^{2}}{2(\delta+m k)(\delta+m k+k)}\left\{\frac{c_{1}^{2} L_{2}}{2}+\left(c_{2}-\frac{c_{1}^{2}}{2}\right) L_{1}+\frac{m b c_{1}^{2} L_{1}^{2}}{2}\right\} .
\end{gathered}
$$

For any complex number $\mu$, we have

$$
\begin{aligned}
a_{m+2}-\mu a_{m+1}^{2}= & \frac{m b k^{2}}{2(\delta+m k)(\delta+m k+k)}\left\{\frac{c_{1}^{2} L_{2}}{2}+\left(c_{2}-\frac{c_{1}^{2}}{2}\right) L_{1}+\frac{m b c_{1}^{2} L_{1}^{2}}{2}\right\} \\
& -\mu \frac{m^{2} b^{2} c_{1}^{2} L_{1}^{2} k^{2}}{4(\delta+m k)^{2}} .
\end{aligned}
$$

Then (55) can be written as

$$
a_{m+2}-\mu a_{m+1}^{2}=\frac{m b L_{1} k^{2}}{2(\delta+m k)(\delta+m k+k)}\left\{c_{2}-v c_{1}^{2}\right\}
$$

where

$$
v=\frac{1}{2}\left\{1-\frac{L_{2}}{L_{1}}-m b L_{1}\left(1-\frac{\delta+m k+k}{\delta+m k} \mu\right)\right\} .
$$

Now, taking absolute value on both sides and using Lemma 3, we obtain the required result.

Putting $m=1$ in Theorem 12, we obtain the following corollary.

Corollary 13. Let $f \in \beta-\mathcal{S T}(\delta, k, b)$ with the form (1). Then, for a complex number $\mu$, we have

$$
\left|a_{3}-\mu a_{2}^{2}\right| \leq \frac{b L_{1} k^{2}}{(\delta+k)(\delta+2 k)} \max \left\{1,\left|\frac{L_{2}}{L_{1}}+b L_{1}\left(1-\frac{\delta+2 k}{\delta+k} \mu\right)\right|\right\} .
$$

\section{Theorem 14. Let}

$$
\begin{aligned}
\sigma_{1} & =\frac{\left\{m b L_{1}^{2}+L_{2}-L_{1}\right\}(\delta+m k)}{m b(\delta+m k+k) L_{1}^{2}}, \sigma_{2}=\frac{\left\{m b L_{1}^{2}+L_{2}+L_{1}\right\}(\delta+m k)}{m b(\delta+m k+k) L_{1}^{2}}, \sigma_{3} \\
& =\frac{\left\{m b L_{1}^{2}+L_{2}\right\}(\delta+m k)}{m b(\delta+m k+k) L_{1}^{2}} .
\end{aligned}
$$
then

If $f$ given by (1) belongs to $\beta-\delta \mathscr{T}_{m}(\delta, k, b)$ with $b>0$,

$$
\begin{aligned}
& \left|a_{m+2}-\mu a_{m+1}^{2}\right| \\
& \leq \begin{cases}\frac{m b L_{1} k^{2}}{(\delta+m k)(\delta+m k+k)}\left\{\frac{L_{2}}{L_{1}}+m b L_{1}\left(1-\frac{\delta+m k+k}{\delta+m k} \mu\right)\right\} & \left(\mu \leq \sigma_{1}\right), \\
\frac{m b L_{1} k^{2}}{(\delta+m k)(\delta+m k+k)} & \left(\sigma_{1} \leq \mu \leq \sigma_{2}\right), \\
-\frac{m b L_{1} k^{2}}{(\delta+m k)(\delta+m k+k)}\left\{\frac{L_{2}}{L_{1}}+m b L_{1}\left(1-\frac{\delta+m k+k}{\delta+m k} \mu\right)\right\} & \left(\mu \geq \sigma_{2}\right) .\end{cases}
\end{aligned}
$$


Further, if $\sigma_{1} \leq \mu \leq \sigma_{3}$, then

$$
\begin{aligned}
& \left|a_{m+2}-\mu a_{m+1}^{2}\right|+\frac{\delta+m k}{m b(\delta+m k+k) L_{1}}\left\{1-\frac{L_{2}}{L_{1}}-m b L_{1}\left(1-\frac{\delta+m k+k}{\delta+m k} \mu\right)\right\}\left|a_{m+1}\right|^{2} \\
& \quad \leq \frac{m b L_{1} k^{2}}{(\delta+m k)(\delta+m k+k)} .
\end{aligned}
$$

$$
\begin{aligned}
& \text { If } \sigma_{3} \leq \mu \leq \sigma_{2} \text {, then } \\
& \begin{aligned}
\mid a_{m+2} & -\mu a_{m+1}^{2} \mid+\frac{\delta+m k}{m b(\delta+m k+k) L_{1}} \\
& \times\left\{1+\frac{L_{2}}{L_{1}}+m b L_{1}\left(1-\frac{\delta+m k+k}{\delta+m k} \mu\right)\right\}\left|a_{m+1}\right|^{2} \\
\leq & \frac{m b L_{1} k^{2}}{(\delta+m k)(\delta+m k+k)} .
\end{aligned}
\end{aligned}
$$

Proof. Applying Lemma 4 to (56) and (57), respectively, we can obtain our results asserted by Theorem 14 .

Putting $m=1$ in Theorem 14, we obtain

\section{Corollary 15. Let}

$$
\begin{aligned}
\sigma_{4} & =\frac{\left\{b L_{1}^{2}+L_{2}-L_{1}\right\}(\delta+k)}{b(\delta+2 k) L_{1}^{2}}, \sigma_{5}=\frac{\left\{b L_{1}^{2}+L_{2}+L_{1}\right\}(\delta+k)}{b(\delta+2 k) L_{1}^{2}}, \sigma_{6} \\
& =\frac{\left\{b L_{1}^{2}+L_{2}\right\}(\delta+k)}{b(\delta+2 k) L_{1}^{2}} .
\end{aligned}
$$
then

If $f$ given by (1) belongs to $\beta-\delta \mathcal{T}(\delta, k, b)$ with $b>0$,

$$
\left|a_{3}-\mu a_{2}^{2}\right| \leq \begin{cases}\frac{b L_{1} k^{2}}{(\delta+k)(\delta+2 k)}\left\{\frac{L_{2}}{L_{1}}+b L_{1}\left(1-\frac{\delta+2 k}{\delta+k} \mu\right)\right\} & \left(\mu \leq \sigma_{4}\right), \\ \frac{b L_{1} k^{2}}{(\delta+k)(\delta+2 k)} & \left(\sigma_{4} \leq \mu \leq \sigma_{5}\right), \\ -\frac{b L_{1} k^{2}}{(\delta+k)(\delta+2 k)}\left\{\frac{L_{2}}{L_{1}}+b L_{1}\left(1-\frac{\delta+2 k}{\delta+k} \mu\right)\right\} & \left(\mu \geq \sigma_{5}\right) .\end{cases}
$$

Further, if $\sigma_{4} \leq \mu \leq \sigma_{6}$, then

$$
\begin{aligned}
& \left|a_{3}-\mu a_{2}^{2}\right|+\frac{\delta+k}{b(\delta+2 k) L_{1}}\left\{1-\frac{L_{2}}{L_{1}}-b L_{1}\left(1-\frac{\delta+2 k}{\delta+k} \mu\right)\right\}\left|a_{2}\right|^{2} \\
& \quad \leq \frac{b L_{1} k^{2}}{(\delta+k)(\delta+2 k)} .
\end{aligned}
$$

$$
\begin{aligned}
& \text { If } \sigma_{6} \leq \mu \leq \sigma_{5} \text {, then } \\
& \begin{array}{l}
\left|a_{3}-\mu a_{2}^{2}\right|+\frac{\delta+k}{b(\delta+2 k) L_{1}}\left\{1+\frac{L_{2}}{L_{1}}+b L_{1}\left(1-\frac{\delta+2 k}{\delta+k} \mu\right)\right\}\left|a_{2}\right|^{2} \\
\quad \leq \frac{b L_{1} k^{2}}{(\delta+k)(\delta+2 k)} .
\end{array}
\end{aligned}
$$

Remark 16. For different choices of the parameters $\beta, m, \delta, k$, and $b$ in the above theorems, we can obtain the corresponding results for each of the following subclasses $\beta-\delta \mathscr{T}_{m}^{\gamma}(\delta$, $k, \alpha), \beta-\delta \mathscr{T}_{m}(\delta, k, \alpha), \beta-\mathcal{S T}_{m}(\delta, b), \beta-\mathcal{S T}_{m}^{\gamma}(\delta, \alpha), \beta$ $-\mathcal{S T}_{m}(\delta, \alpha), \beta-\mathcal{S T}(\delta, b), \beta-\mathcal{S} \mathscr{T}^{\gamma}(\delta, \alpha), \beta-\mathcal{S T}(\delta, \alpha)$, $\beta-\mathcal{S T}^{\gamma}(\delta, k, \alpha), \beta-\mathcal{S T}(\delta, k, \alpha), \beta-\mathcal{S} \mathscr{T}_{m}(b), \beta-\mathcal{S T}_{m}^{\gamma}($ $\alpha), \beta-\mathcal{S T}_{m}(\alpha), \beta-\mathcal{S T}(b), \beta-\mathcal{S T}^{\gamma}(\alpha), \beta-\mathcal{S} \mathscr{T}(\alpha), \mathcal{S}_{m}($ $\delta, k, b), \quad \delta_{m}^{\gamma}(\delta, k, \alpha), \delta_{m}^{\gamma}(\alpha), \delta_{m}(b)$, and $\delta(b)$ which are defined in Section 1.

\section{Data Availability}

No data were used to support this study.

\section{Ethical Approval}

This article does not contain any studies with human participants or animals performed by any of the authors.

\section{Conflicts of Interest}

The authors declare that they have no competing interests.

\section{Authors' Contributions}

All authors contributed equally to the writing of this paper. All authors read and approved the final manuscript.

\section{References}

[1] T. Bulboacă, Differential Subordinations and Superordinations, Recent Results, House of Scientific Book Publ., Cluj-Napoca, 2005.

[2] S. S. Miller and P. T. Mocanu, Differential Subordinations: Theory and Applications, Series on Monographs and Textbooks in Pure and Applied Mathematics, vol. 225, Marcel Dekker, New York and Basel, 2000.

[3] S. S. Miller and P. T. Mocanu, "Subordinants of differential superordinations," Complex Variables, Theory and Application: An International Journal, vol. 48, no. 10, pp. 815-826, 2003.

[4] R. Daz and E. Pariguan, "On hypergeometric functions and Pochhammer $k$-symbol," Divulgaciones Matemáticas, vol. 15, no. 2, pp. 179-192, 2007.

[5] R. M. Goel and N. S. Sohi, "A new criterion for $p$ - valent functions," Proceedings of American Mathematical Society, vol. 78, no. 3, pp. 353-357, 1980.

[6] M. K. Aouf, "A generalization of multivalent functions defined by Ruscheweyh derivatives," Soochow Journal of Mathematics, vol. 17, pp. 83-97, 1991. 
[7] V. Kumar and S. L. Shukla, "Multivalent functions defined by Ruscheweyh derivatives," Indian Journal of Pure and Applied Mathematics, vol. 15, no. 11, pp. 489-509, 1984.

[8] M. K. Aouf and H. E. Darwish, "A remark on certain $p$-valent functions," International Journal of Mathematics and Mathematical Sciences, vol. 19, no. 2, 406 pages, 1996.

[9] T. M. Seoudy, "Some subclasses of univalent functions associated with $k$-Ruscheweyh derivative operator," Ukrainian Mathematical Journal, In press.

[10] S. Ruscheweyh, "New criteria for univalent functions," Proceedings of the American Mathematical Society, vol. 49, no. 1, pp. 109-115, 1975.

[11] S. Kanas and A. Wisniowska, "Conic regions and $k$-uniform convexity," Journal of Computational and Applied Mathematics, vol. 105, no. 1-2, pp. 327-336, 1999.

[12] S. Kanas and A. Wisniowska, "Conic domains and starlike functions," Revue Roumaine de Mathématiques Pures et Appliquées, vol. 45, no. 4, pp. 647-658, 2000.

[13] K. I. Noor, M. Arif, and W. Ul-Haq, "On $k$-uniformly close-toconvex functions of complex order," Applied Mathematics and Computation, vol. 215, no. 2, pp. 629-635, 2009.

[14] T. O. Salim, M. S. Marouf, and J. M. Shenan, "A subclass of multivalent uniformly convex functions associated with generalized Salagean and Ruscheweyh differential operators," Acta Universitatis Apulensis, vol. 26, pp. 289-300, 2011.

[15] R. Bharati, R. Parvatham, and A. Swaminathan, "On subclasses of uniformly convex functions and corresponding class of starlike functions," Tamkang Journal of Mathematics, vol. 28, no. 1, pp. 17-32, 1997.

[16] M. K. Aouf, "On coefficient bounds of a certain class of $p$ -valent $\lambda$-spiral functions of order $\alpha$," International Journal of Mathematics and Mathematical Sciences, vol. 10, no. 2, 266 pages, 1987.

[17] H. M. Srivastava, M. K. Aouf, and S. Owa, "Certain classes of multivalent functions of order $\alpha$ and type $\beta$," Bulletin de la Societe Mathematique de Belgique. Serie B, vol. 42, no. 1 , pp. 31-66, 1990.

[18] B. Wongsaijai and N. Sukantamala, "Mapping properties of generalized $q$-integral operator of $p$-valent functions involving the Ruscheweyh derivative and the generalized Salagean operator," Acta Universitatis Apulensis, vol. 41, pp. 3150, 2015.

[19] M. A. Nasr and M. K. Aouf, "Bounded starlike functions of complex order," Indian Academy of Sciences, vol. 92, no. 2, pp. 97-102, 1983.

[20] M. A. Nasr and M. K. Aouf, "Starlike function of complex order," The Journal of Natural Sciences and Mathematics, vol. 25, no. 1, pp. 1-12, 1985.

[21] S. Kanas, "Coefficient estimates in subclasses of the Carathéodory class related to conical domains," Acta Mathematica Universitatis Comenianae, vol. 75, no. 2, pp. 149-161, 2005.

[22] W. Ma and D. Minda, "A unified treatment of some special classes of univalent functions," Proceedings of the Conference on Complex Analysis (Tianjin, 1992), Z. Li, F. Y. Ren, L. Yang, and S. Y. Zhang, Eds., , pp. 157-169, Int. Press, Cambridge, MA, 1994. 